\title{
Laser-induced plasmas in micromachined fused silica cavities
}

\author{
Xianzhong Zeng, Samuel S. Mao, Chunyi Liu, Xianglei Mao, Ralph Greif, Richard E. Russo* \\ Lawrence Berkeley National Laboratory, Berkeley, CA 94720 \\ rerusso@lbl.gov
}

\begin{abstract}
Cavity formation is a frequent result in many laser ablation applications. Although most theoretical investigations have been devoted to laser ablation on a flat surface, the development of a laser plasma inside a cavity is of both fundamental as well as practical significance. In this study, the temperature and electron number density of laser-induced plasmas in fused silica cavities were determined using spectroscopic methods. The effects of cavity aspect ratio on plasma properties were investigated. The temperature and electron number density of laser-induced plasma were measured to be much higher and to decrease faster for a plasma inside a cavity than on the flat surface. Cavity wall influences on the plasma expansion are discussed.
\end{abstract}

Keywords: laser ablation, cavity structure, laser-induced plasma 
Laser ablation is a powerful technology for direct material analysis, deposition of thin films, rapid prototyping and micromachining, among other applications. Coupling intense electromagnetic energy of a laser pulse with a target material can result in melting, vaporization, ejection of atoms, ions, and molecular species, as well as shock wave formation and plasma development [1]. These processes for mass removal are dependent on the laser and material properties. In many laser-material interaction applications such as micro-machining and solid sampling for chemical analysis, cavity structures with specific aspect ratios (depth-to-diameter ratio) develop after repetitive pulses irradiate the same target location. As a consequence, the laser interaction is with the resulting cavity structure, rather than the flat surface.

The amount of energy absorbed by a target material (copper and aluminum) was found to increase with increasing aspect ratio, according to thermal radiation analysis [2]. For aspect ratios varying from 0 to 5 , the amount of energy absorbed could differ from 1 to 10 times the energy absorbed by a flat surface. Numerical calculations [3] demonstrated how a large amount of the reflected laser beam could be absorbed by the cavity wall, enhancing the energy coupling between the laser beam and the target. The cavity aspect ratio was found to be an important parameter influencing the degree of elemental fractionation for chemical analysis. Fractionation represents the phenomenon in which the laser-ablated mass (vaporized and ionized) has a different chemical composition than that of the target material. Fractionation was found to be significant when the cavity aspect ratio exceeded approximately 6.0 [4]. Despite theoretical implications of increased laser energy coupling to a solid target when high aspect ratio cavities are present, the fundamental processes of laser interactions with a cavity 
structure remains unexplored. The scope of this research was to investigate laser ablation inside cavity structures, in particular, to measure the spectral characteristics of laser ablation plasmas developed inside and outside cavities of different aspect ratios.

Fused silica glass was selected as the target material, and silicon emission lines were measured to determine the plasma properties (plasma temperature and electron number density). Cavities of different diameters and depths were fabricated using a laser ablation/micromachining workstation. The experimental system included a quadrupled Nd:YAG laser operating at $266 \mathrm{~nm}$ with a 3-ns pulse duration. The laser irradiance used for these experiments was $\sim 7 \mathrm{GW} / \mathrm{cm}^{2}$. Cavities were pre-fabricated and then single pulse laser ablation was performed at the bottom surface of these cavities. The laser beam was focused onto the target to a 50-micron spot diameter with a quartz lens. A second lens was used to image the laser-induced plasma into the entrance slit of a spectrometer. The emission spectra were detected by an Intensified Charge-Coupled Device (ICCD) system consisting of a thermoelectrically cooled CCD with a microchannel plate image intensifier. This spectrometer and detection system provides $0.1 \mathrm{~nm}$ spectral resolution. Dark current background of the CCD detector was subtracted from the measured spectral data for each measurement. Spatial distribution of plasma temperature and electron number density were determined by measuring Stark broadening of the silicon emission lines and the relative line-continuum ratio $[5,6]$. The emphasis of this study was spatially and temporally resolved measurements during the early phase $(<300 \mathrm{~ns})$ of the laser plasma generated inside cavity structures. 
Fig. 1 shows the plasma spectra from laser ablation inside a 160-micron diameter, 480-micron deep cavity (Fig. 1a), compared with the plasma spectra from laser ablation on the flat surface (Fig. 1b). The laser irradiance was approximately $16 \mathrm{GW} / \mathrm{cm}^{2}$ at both solid surfaces. The silicon emission line $\mathrm{Si}(\mathrm{I})$ at $288.16 \mathrm{~nm}$ was used for plasma diagnostics. The spectra inside the cavity have a wider Full Width at Half Maximum (FWHM) as well as a higher emission intensity. The position of maximum emission intensity is at $0.9 \mathrm{~mm}$ from the bottom of the cavity; for flat surface results, the location of maximum emission intensity occurs at $0.6 \mathrm{~mm}$ above the surface.

Spectral line profiles are due mainly to Stark broadening, which results from collisions of charged species. Values of FWHM $\Delta \lambda_{1 / 2}$ are obtained by fitting the observed line profiles using a Lorentzian function. The FWHM of Stark broadened lines is related to the electron number density by the expression [8],

$$
\Delta \lambda_{1 / 2}=2 W\left(\frac{n_{e}}{10^{16}}\right)\left[1+1.75 A\left(\frac{n_{e}}{10^{16}}\right)^{1 / 4}\left(1-\frac{3}{4} N_{D}^{-1 / 3}\right)\right],
$$

where $n_{e}$ is the electron number density in $\mathrm{cm}^{-3}, \Delta \lambda_{1 / 2}$ is in $\mathrm{nm} . N_{D}$ is the number of particles in the Debye sphere, $N_{D}=1.72 \times 10^{9} T^{3 / 2} / n_{e}{ }^{1 / 2}$, with $T$ in eV and $n_{e}$ in $\mathrm{cm}^{-3}$. Under the assumption of local thermal equilibrium (LTE), the plasma temperature $T$ (in $\mathrm{K})$ can be determined by the relative line-to-continuum intensity ratio using the following expression [6-8],

$$
\frac{\varepsilon_{l}}{\varepsilon_{c}}(\lambda)=C_{r} \frac{A_{21} g_{2}}{U_{i}} \frac{\lambda_{c}^{2}}{\lambda_{l} T} \frac{\exp \left(\frac{E_{i}-E_{2}-\Delta E_{i}}{k T}\right)}{\left[\xi\left(1-\exp \frac{-h c}{\lambda k T}\right)+G\left(\exp \frac{-h c}{\lambda k T}\right)\right]}
$$


where $\varepsilon_{c}$ is the continuum emission coefficient; $\varepsilon_{l}$ is the integrated emission coefficient over the line spectral profile. The parameters used for $T_{e}$ and $n_{e}$ calculations can be found in reference [9].

A necessary condition for the LTE assumption is $n_{e}\left(\mathrm{~cm}^{-3}\right) \geq 1.4 \times 10^{14} T^{1 / 2} \Delta E^{3}$ [10]. For the $\mathrm{Si}(\mathrm{I})$ transition at $288.16 \mathrm{~nm}, \Delta E=4.3 \mathrm{eV}$. At the highest temperature of about $10 \mathrm{eV}$, the lower limit for $n_{e}$ is smaller than $10^{17} \mathrm{~cm}^{-3}$, which is more than one order of magnitude lower than the value of $n_{e}$ deduced from Stark broadening. Therefore, the validity of the LTE assumption is supported.

Figure 2 shows the plasma temperatures $T$ and electron number densities $n_{e}$ for laser ablation of three cavities as well as the flat surface. These three cavities have the same depth $(\sim 480$ micron) but different diameters $(80,160$, and $490 \mu \mathrm{m})$. The plasma temperature and electron number density are highest in the largest aspect-ratio cavity. As the aspect ratio decreases, the plasma temperature and electron number density in the cavity approach the flat surface condition. For the largest aspect ratio, the plasma temperature decreases from $38000 \mathrm{~K}$ (in the cavity) to $30000 \mathrm{~K}$ ( $1.5 \mathrm{~mm}$ above the cavity bottom); electron number density falls from $2 \times 10^{19}$ to $5 \times 10^{18} \mathrm{~cm}^{-3}$. On a flat surface, the plasma temperature and electron number density are much lower, $20000 \mathrm{~K}$ and $2 \times 10^{18}$ $\mathrm{cm}^{-3}$, respectively, and do not change as much with distance from the surface.

These results are believed to be related to reflection and confinement effects by the cavity walls and plasma shielding (absorption and/or reflection of the laser beam by the plasma). 
Once the plasma is initiated in the cavity, its lateral expansion is confined; electron number density increases greatly. The cavity acts as a trap in which light energy of the trailing edge of the laser pulse can be efficiently absorbed by the confined laser plasma. Additional electrons will be produced via electron-neutral, electron-ion inverse bremsstrahlung, and photoionization. After the plasma is significantly ionized, electronion inverse bremsstrahlung dominates the absorption. Given the laser wavelength and plasma density measured, the absorption coefficient for electron-ion inverse bremsstrahlung was estimated to be $\sim 1 \mathrm{~cm}^{-1}$ at time of $3 \mathrm{~ns}$ for the aspect ratio $\sim 3$ cavity [9]. The absorbed energy is converted into internal energy of the plasma. After the completion of the laser pulse, the plasma expands out of the cavity and the plasma temperature and electron number density in the cavity decrease.

Two locations were selected for the cavity and one for the flat surface to compare plasma temperature and electron number density as a function of time (Fig. 3). Location A is inside the cavity at a height of $0.2 \mathrm{~mm}$ above the bottom surface, location B is outside of the cavity at a height of $0.7 \mathrm{~mm}$ above the bottom of the cavity. Location $\mathrm{C}$ is $0.2 \mathrm{~mm}$ above the flat surface. Location $\mathrm{A}$ and $\mathrm{C}$ are equal distance above solid material.

An adiabatic model is often used to describe laser-induced plasma expansion [12]. For an adiabatic expansion, the expressions for plasma temperature and electron number density as a function of time are [9] $T(t) \propto t^{-2 \alpha(\gamma-1) /[\alpha(\gamma-1)+2]}$ and $n_{e}(t) \propto t^{-2 \alpha /[\alpha(\gamma-1)+2]}$, where $\gamma$ is the ratio of specific heat capacities at constant pressure to constant volume, and $\alpha$ is the flow dimensionality: $1<\alpha<3$ [12]. 
The plasma at location $\mathrm{C}$ from laser ablation on the flat surface gives:

$T \propto t^{-0.54 \pm 0.04}, n_{e} \propto t^{-0.95 \pm 0.06}$, solving for $\alpha$ and $\gamma$ yields: $\alpha=1.30 \pm 0.12, \gamma=1.57 \pm 0.08$.

The calculated values of $\alpha$ and $\gamma$ are within experimental error to the values obtained by Liu, et al. [9]. The calculated dimensionality 1.30 supports preferential spatial expansion in the direction perpendicular to the target. The calculated $\gamma$ value, 1.57 is close to the specific heat for an ideal gas of $5 / 3$. Therefore, an adiabatic process can be used to describe the plasma expansion for this experiment.

For the plasma that has expanded outside of the cavity at location B:

$T \propto t^{-0.52 \pm 0.03}, n_{e} \propto t^{-0.98 \pm 0.05}$, corresponding to: $\alpha=1.32 \pm 0.09, \gamma=1.53 \pm 0.06$, which are close to the flat surface results above. Thus, after the plasma expands out of the cavity, an adiabatic model can describe plasma temperature and electron number density variations.

For the plasma in the cavity at $\mathrm{A}, T \propto t^{-1.08 \pm 0.04}, n_{e} \propto t^{-1.51 \pm 0.06}$. Therefore, $\alpha=3.31 \pm 0.17, \gamma=1.72 \pm 0.05$. The dimensionality value inside the cavity is much greater than outside of the cavity, which indicates the cavity wall influences on the plasma expansion. The influences include confinement effects by the cavity walls and energy transfer from plasma to the walls. Once the plasma is generated, hydrodynamic confinement of the plasma expansion occurs in the cavity; energy from the plasma can be transferred to the walls by normal electron heat conduction, electron-ion (exothermic) recombination on the cavity walls, short-wavelength thermal plasma radiation, and condensation of vapor that moves to the surface due to the plasma pressure. The plasma 
cools rapidly by interaction with cavity walls, resulting in a sharp decrease of plasma temperature. In addition, the confinement effects may modify the shock wave propagation inside the cavity, influencing laser beam interactions with the hydrodynamic expansion of the plasma.

\section{Acknowledgments}

This research was supported by the U.S. Department of Energy, Office of Basic Energy Sciences, Chemical Sciences Division, under contract No. DE-AC03-76SF00098. 


\section{References}

[1] R.E. Russo, X. Mao, and S.S. Mao, Analytical Chemistry, 74, 70A (2002).

[2] M. A. Shannon, Appl. Surf. Sci., 129, 218 (1998).

[3] S.H. Jeong, R. Greif, and R.E. Russo, J. Appl. Phys., 80, 1996 (1996).

[4] A.J.G. Mank and P.D. Mason, J. Anal. At. Spectrom., 14, 1143 (1999).

[5] H.R. Griem, Spectral Line Broadening by Plasmas (Academic, New York, 1974).

[6] H.R. Griem, Principles of Plasma Spectroscopy (Cambridge University Press, 1997).

[7] W. Demtroder, Laser Spectroscopy (Springer, New York, 1998).

[8] G. Befeki, Principles of laser plasmas (Wiley Interscience, New York, 1976).

[9] H.C. Liu, X. Mao, J.H. Yoo, and R.E. Russo, Spectrochimica Acta, B 54, 1607 (1999).

[10] P.J. Wolf, J. Appl. Phys., 72, 1280 (1992)

[11] M.V. Allmen, Laser-beam Interactions with Materials (Springer-Verlag, New York, 1987).

[12] R.K. Singh, O.W. Holland, J. Narayan, J. Appl. Phys. 68, 233 (1998). 


\section{Figure Captions}

Figure 1. (a) Spatial distribution of plasma emission from the 160-micron diameter cavity (depth 480 micron) at the center wavelength of $288.16 \mathrm{~nm}$ and time of $100 \mathrm{~ns}$. Cavity bottom is at $-0.5 \mathrm{~mm}$. (b) Spatial distribution of plasma emission from the flat surface at the center wavelength of $288.16 \mathrm{~nm}$ and time of $100 \mathrm{~ns}$.

Figure 2. (a) Plasma temperature and (b) electron number density vs. distance for different aspect ratio cavities (delay $=100 \mathrm{~ns}$, irradiance $7.0 \mathrm{GW} / \mathrm{cm}^{2}$ ). Aspect ratio $=$ Depth / Diameter $=\mathrm{L}$ (micron) $/ \mathrm{D}$ (micron). L / $\mathrm{D}_{1}=480 / 80 \sim 6 ; \mathrm{L} / \mathrm{D}_{2}=480 / 160 \sim$ $3 ; \mathrm{L} / \mathrm{D}_{3}=480 / 490 \sim 1$.

Figure 3. Temporal evolution of plasma temperature (a) and electron number densities (b) inside and outside of the cavity. Cavity diameter is 80 micron and depth is 480 micron. Irradiance is $7.6 \mathrm{GW} / \mathrm{cm}^{2}$. A) Plasma inside cavity, distance $\mathrm{d}=0.2 \mathrm{~mm}$. B) Plasma outside of cavity, $d=0.7 \mathrm{~mm}$. C) Plasma from flat surface, $d=0.2 \mathrm{~mm}$. 


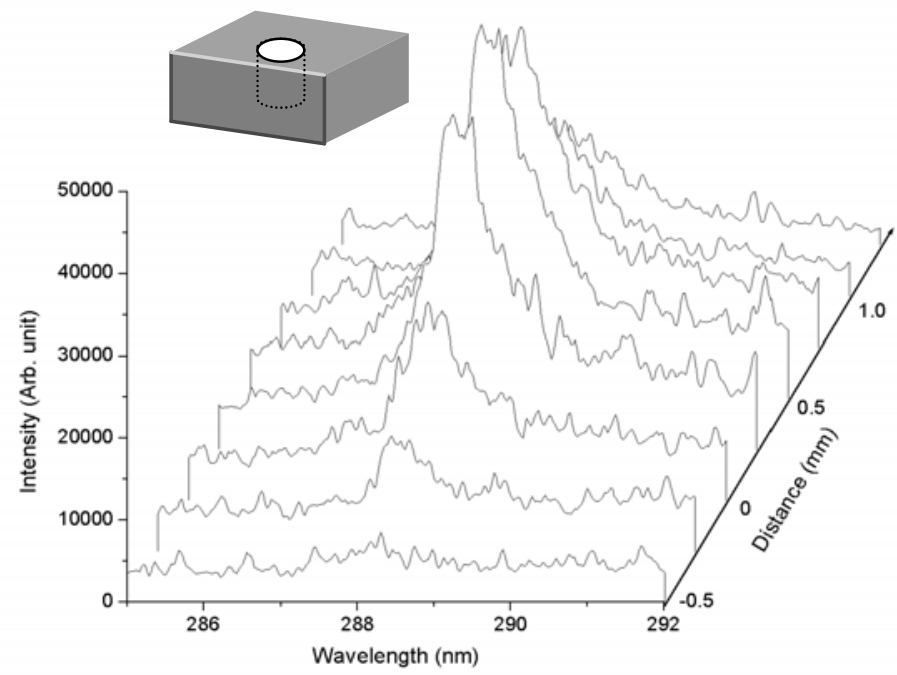

a

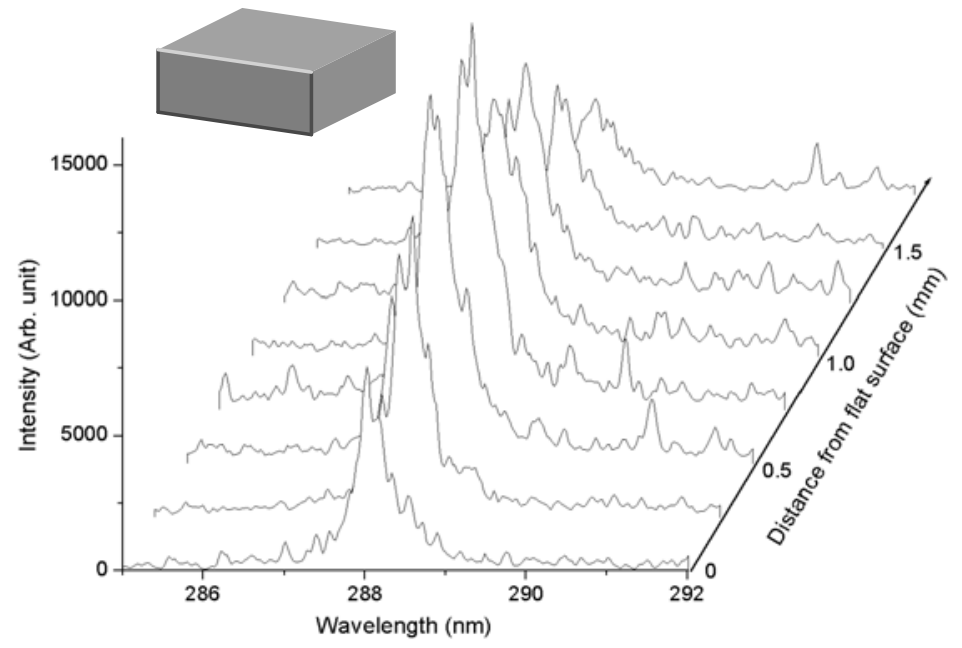

b

Figure 1 


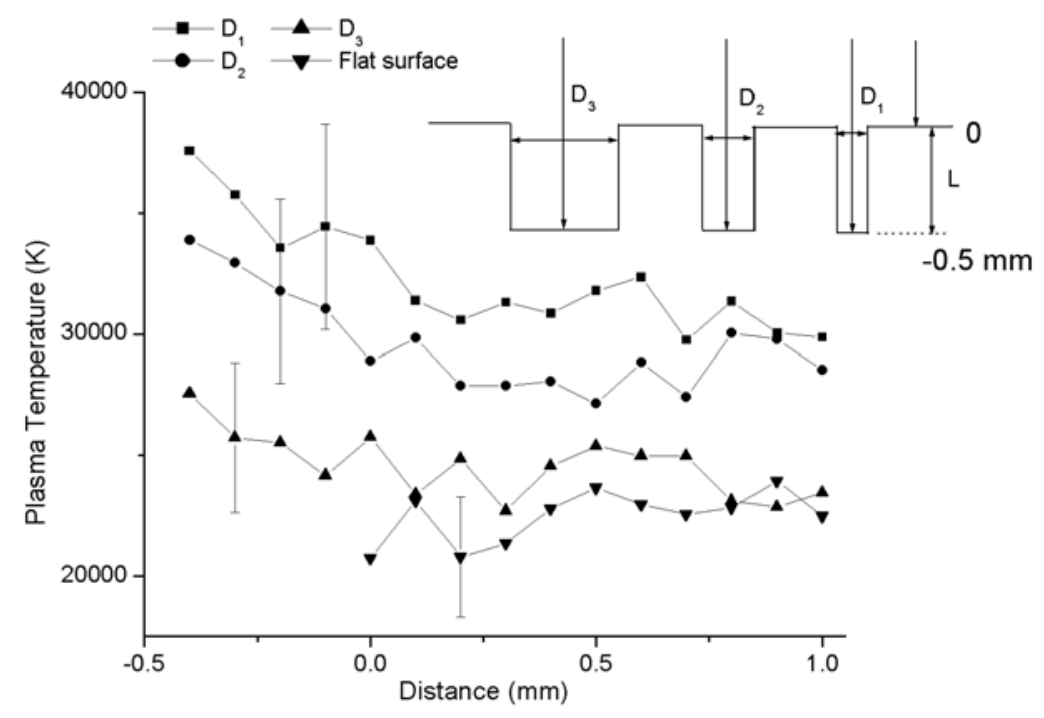

a

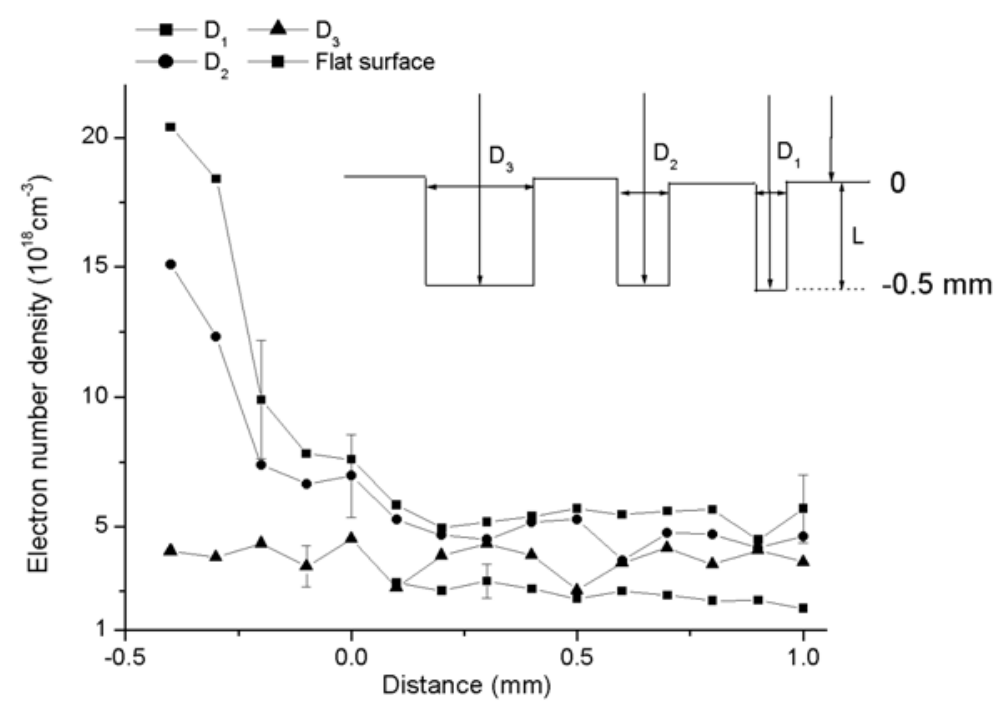

b

Figure 2 


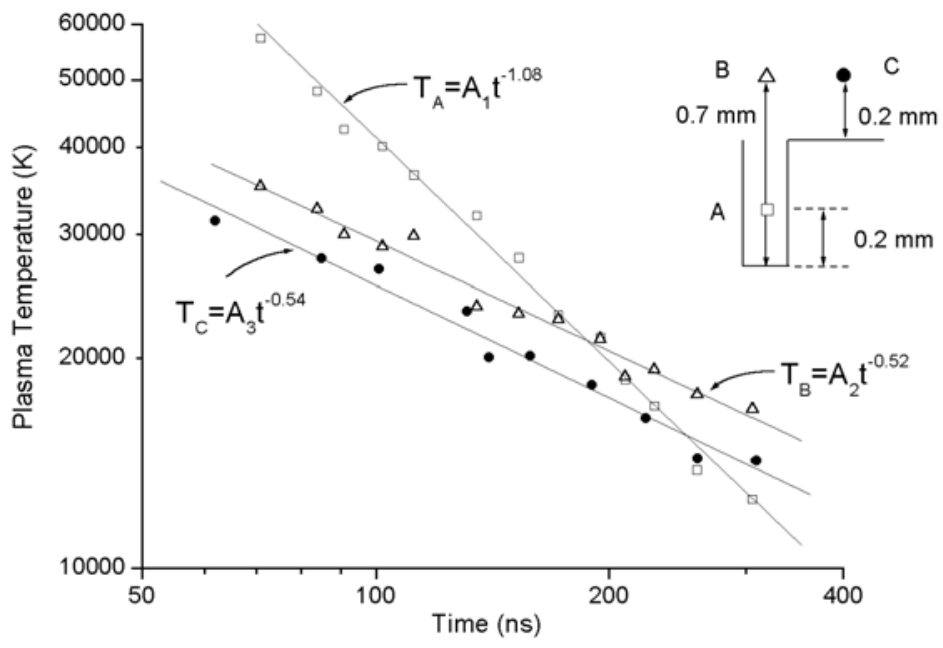

a

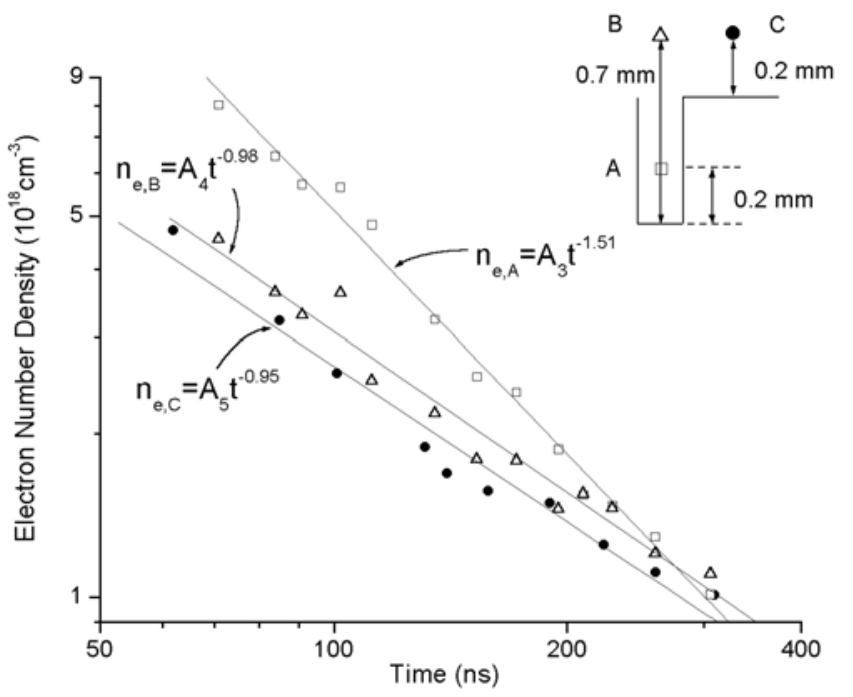

b

Figure 3 